\title{
THE COMMUTANT OF ANALYTIC TOEPLITZ OPERATORS
}

BY

\author{
JAMES A. DEDDENS(1) AND TIN KIN WONG
}

\begin{abstract}
In this paper we study the commutant of an analytic Toeplitz operator. For $\phi \in H^{\infty}$, let $\phi=\chi F$ be its inner-outer factorization. Our main result is that if there exists $\lambda \in C$ such that $x$ factors as $x=x_{1} x_{2} \cdots x_{n}$, , each $x_{i}$ an inner function, and if $F-\lambda$ is divisible by each $x_{i}$, then $\left\{T_{\phi}\right\}^{\prime}=$ $\left\{T_{\chi}\right\}^{\prime} \cap\left\{T_{F}\right\}^{\prime}$. The key step in the proof is Lemma 2, which is a curious result about nilpotent operators. One corollary of our main result is that if $\lambda(z)=z^{n}, n \geq 1$, then $\left\{T_{\phi}\right\}^{\prime}=\left\{T_{\chi}\right\}^{\prime} \cap\left\{T_{F}\right\}^{\prime}$, another is that if $\phi \in H^{\infty}$ is univalent then $\left\{T_{\phi}\right\}^{\prime}=\left\{T_{z}\right\}^{\prime}$. We are also able to prove that if the inner factor of $\phi$ is $x(z)=z n, n \geq 1$, then $\left\{T_{\phi}\right\}^{\prime}=\left\{T_{z^{s}}\right\}^{\prime}$ where $s$ is a positive integer maximal with respect to the property that $z^{n}$ and $F(z)$ are both functions of $z^{s}$. We conclude by raising six questions.
\end{abstract}

1. Introduction. Let $H^{2}$ denote the Hilbert space of functions $f$ analytic in the open unit disc $\mathbf{D}$ for which the functions $f_{r}(\theta)=f\left(r e^{i \theta}\right)$ are uniformly bounded in $L^{2}$-norm for $r<1$, and let $H^{\infty}$ denote the linear manifold of bounded functions in $H^{2}$. For $\phi \in H^{\infty}, T_{\phi}$ (or $T_{\phi(z)}$ ) is the analytic Toeplitz operator on $H^{2}$ defined by the relation $\left(T_{\phi} f\right)(z)=\phi(z) f(z)$. These operators have received a great deal of attention recently and many of their properties are well known ([4], [5]). The operator $T_{z}$ is often called the unilateral shift and is the canonical example of a completely nonunitary isometry of defect one. Every analytic Toeplitz operator commutes with $T_{z}$, in fact, every operator that commutes with $T_{z}$ is an analytic Toeplitz operator. The purpose of this paper is to study the commutant of an arbitrary analytic Toeplitz operator. We obta in some partial results characterizing the commutant of an analytic Toeplitz operator as well as some partial results characterizing those analytic functions whose associated Toeplitz operators have commutant equal to that of $T_{z}$. $\$ 2$ contains our main result stated in terms of pure isometries, $\$ \S 3$ and 4 contain numerous results on the commutant of analytic Toeplitz operators, while $\$ 5$ contains some open questions.

It is well known that if $f \in H^{2}$ then there is a function $f^{*} \in L^{2}(\mathrm{~T})$ such that $f\left(r e^{i \theta}\right)$ converges almost everywhere to $f^{*}\left(e^{i \theta}\right) . \chi \in H^{\infty}$ is said to be an inner

Received by the editors July 31, 1972 and, in revised form, February 28, 1973. AMS (MOS) subject classifications (1970). Primary 47B35, 47B20, 30A76.

Key words and phrases. Analytic function, inner and outer functions, $H^{\infty}, H^{2}$, analytic Toeplitz operator, pure is ometry, commutant.

(1) Research supported by University of Kansas General Research Fund. 
function if $\left|\chi^{*}\left(e^{i \theta}\right)\right|=1$ almost everywhere (or equivalently, if $T_{\chi}$ is an isometry). Every inner function $\chi$ has a factorization $\chi(z)=e^{i \gamma} B(z) S(z)$ with $\left|e^{i \gamma}\right|=1$ where $B(z)$ is a Blaschke product of the form

$$
B(z)=z^{n} \prod_{k=1}^{\infty} \frac{\left|a_{k}\right|}{a_{k}} \frac{a_{k}-z}{1-\bar{\alpha}_{k} z}, \quad 0<\left|a_{k}\right|<1,
$$

and $S(z)$ is a singular inner function of the form

$$
S(z)=\exp \left\{-\int_{0}^{2 \pi} \frac{e^{i t}+z}{e^{i t}-z} d \mu(t)\right\}
$$

with $\mu$ a singular measure. $F \in H^{\infty}$ is said to be an outer function if $F$ is of the form

$$
F(z)=\exp \left\{\frac{1}{2 \pi} \int_{0}^{2 \pi} \frac{e^{i t}+z}{e^{i t}-z} k(t) d t\right\}
$$

where $k$ is a real-valued integrable function (or equivalently, if $T_{F}$ has dense range). Every nonconstant function $\phi \in H^{\infty}$ has a unique factorization of the form $\phi(z)=\chi(z) F(z)$ where $\chi \in H^{\infty}$ is an inner function and $F \in H^{\infty}$ is an outer function ([6], [10]). Our results will show that this factorization plays a key role in determining the commutant of $T_{\phi}$.

Although we are primarily interested in a nalytic Toeplitz operators it will be convenient to state some of our results more generally. An isometry $V$ on a Hilbert space $\mathcal{H}$ is called a pure isometry $([3],[7])$ if $\bigcap_{n=0}^{\infty} V^{n} \mathcal{H}=\{0\}$. The dimension of the defect space $K_{V}=\mathcal{H} \ominus V \mathcal{H}$ is called the defect or multiplicity of $V$, and one easily obtains the decomposition $\mathcal{H}=\sum_{n=0}^{\infty} \oplus V^{n} K_{V}$. Any two pure isometries of the same multiplicity are unitarily equivalent. Thus $V$ is unitarily equivalent to the unilateral shift $U_{+}$on $l_{+}^{2}\left(K_{V}\right)=\Sigma_{n=0}^{\infty} \bigoplus K_{V}$ defined by

$$
U_{+}\left(x_{0}, x_{1}, x_{2}, \ldots\right)=\left(0, x_{0}, x_{1}, x_{2}, \ldots\right) \text {. }
$$

It is easily verified that the commutant of $U_{+}$consists of those bounded linear operators on $l_{+}^{2}\left(K_{V}\right)$ of the form $\sum_{n=0}^{\infty} \hat{A}_{n} U_{+}^{n}$ where, for $A$ a bounded linear operator on $K_{V}, \hat{A}$ (the inflation of $A$ ) is defined on $l_{+}^{2}\left(K_{V}\right)$ by

$$
\hat{A}\left(x_{0}, x_{1}, x_{2}, \ldots\right)=\left(A x_{0}, A x_{1}, A x_{2}, \ldots\right) \text {. }
$$

Thus the commutant of a pure isometry can be characterized.

If $\chi \in H^{\infty}$ is a nonconstant inner function then $T_{\chi}$ is a pure isometry (this follows since no $0 \neq f \in H^{\infty}$ is infinitely divisible by $\chi$ ), which has finite defect if and only if $\chi$ is a finite Blaschke product. Hence the commutant of $T_{\chi}$ for $\chi$ an inner function is well known, and we attempt to characterize the commutant of 
an arbitrary analytic Toeplitz operator in terms of these objects. For a Hilbert space $\mathcal{H}, B(\mathcal{H})$ will denote the algebra of all bounded linear operators on $\mathcal{H}$. If $A \in \mathcal{B O}()$ then $\{A\}^{\prime}$ will denote the commutant of $A$, that is, $\{A\}^{\prime}=\{B \in \mathcal{B}(\mathcal{H})$ : $A B=B A\}$.

2. Main result. Throughout this section $K$ will denote a Hilbert space, $\mathcal{H}$ will denote the Hilbert space $l_{+}^{2}(K)$, and $U_{+}$will denote the unilateral shift on H.

Lemma 1. Suppose $S \in \mathcal{B O (})$ bas dense range and commutes with $U_{+}$. If $T \in \mathbb{B}(\mathcal{H})$ commutes with $S U_{+}$then $T$ bas a lower triangular operator-valued matrix on $\mathcal{H}$.

Proof. Since $T$ lower triangular is equivalent to $T^{*}$ upper triangular, which in turn, is equivalent to the subs paces $M_{n}=\Sigma_{k=0}^{n} \oplus K$ invariant for $T^{*}$, it suffices to prove that $T^{*}$ leaves $M_{n}$ invariant. Since $T$ commutes with $S U_{+} T^{*}$ commutes with $S^{*} U_{+}^{*}$ and hence with $S^{* n+1} U_{+}^{* n+1}$. Thus $T^{*}$ leaves invariant the null space of $S^{* n+1} U_{+}^{* n+1}$. Because $S$ has dense range, $S^{* n+1}$ is one-toone, and

$$
\operatorname{null}\left(S^{* n+1} U_{+}^{* n+1}\right)=\operatorname{null}\left(U_{+}^{* n+1}\right)=M_{n}
$$

Hence $T$ is lower triangular.

The following lemma is essential for our main result.

Lemma 2. Let $N$ be a nilpotent operator on $K$ and let $X_{0}=\lambda I+N$ where $0 \neq \lambda \in \mathbf{C}$. If $B, A_{0}, A_{1}, A_{2}, \cdots \in \mathfrak{B}(K)$ satisfy

(a) $\left\|A_{k}\right\| \leq M, k=0,1,2, \cdots$, and

(b) $A_{k} X_{0}=X_{0} A_{k-1}+B, k=1,2,3, \ldots$, then $A_{0}=A_{1}=A_{2}=\cdots$.

Proof. By Theorem 1 in [8], $K$ decomposes into $\Sigma_{i=1}^{n} \dot{\oplus} K_{i}$ and $x_{0}$ has a lower triangular operator-valued matrix with diagonal elements $\lambda I_{i}$. We show that $A_{0}=A_{1}=A_{2}=\cdots$ by showing that they must have the same $(1, n),(1, n-1)$, $\ldots,(1,1),(2, n), \ldots,(2,1), \ldots,(n, n), \ldots,(n, 1)$ operator entries with respect to o this decomposition. We repeatedly use the obvious fact that the lemma is true if $N=0$. More precisely, if $D, C_{0}, C_{1}, C_{2}, \cdots \in \mathfrak{B}\left(K^{\prime}, K^{\prime \prime}\right)$ satisfying $\left\|C_{k}\right\| \leq M$ and $\lambda C_{k}=\lambda C_{k-1}+D$ for $k=1,2,3, \ldots$ then $C_{0}=C_{1}=C_{2}=\ldots$. To see that this is the case merely observe that $\lambda C_{k}=\lambda C_{k-1}+D$ implies $\lambda C_{k}=\lambda C_{0}+k D$. In order that $\left\|C_{k}\right\| \leq M$ we must have that $D=0$ and hence $C_{0}=C_{1}=C_{2}=\cdots$.

Now the $(1, n)$ entry of $(b)$ is

$$
\lambda\left(A_{k}\right)_{1, n}=\lambda\left(A_{k-1}\right)_{1, n}+(B)_{1, n}, \quad k=0,1,2, \ldots,
$$


and so by the above remark and by the fact that (a) implies $\left\|\left(A_{k}\right)_{i, j}\right\| \leq M$ for $i, j=1,2, \cdots, n, k=0,1,2, \ldots$ one concludes that $\left(A_{0}\right)_{1, n}=\left(A_{1}\right)_{1, n}=$ $\left(A_{2}\right)_{1, n}=\cdots$. The $(1, n-1)$ entry of $(b)$ is

$$
\lambda\left(A_{k}\right)_{1, n-1}+\left(X_{0}\right)_{n, n-1}\left(A_{k}\right)_{1, n}=\lambda\left(A_{k-1}\right)_{1, n-1}+(B)_{1, n-1}
$$

or since $\left(A_{0}\right)_{1, n}=\left(A_{1}\right)_{1, n}=\cdots$, that

$$
\lambda\left(A_{k}\right)_{1, n-1}=\lambda\left(A_{k-1}\right)_{1, n-1}+\left[(B)_{1, n-1}-\left(X_{0}\right)_{n, n-1}\left(A_{0}\right)_{1, n}\right]
$$

and again we conclude that $\left(A_{0}\right)_{1, n-1}=\left(A_{1}\right)_{1, n-1}=\cdots$.

Now the $(i, j)$ entry of $(b)$ is

$$
\begin{aligned}
\lambda\left(A_{k}\right)_{i, j} & +\left(A_{k}\right)_{i, j+1}\left(X_{0}\right)_{j+1, j}+\cdots+\left(A_{k}\right)_{i, n}\left(X_{0}\right)_{n, j} \\
& =\lambda\left(A_{k-1}\right)_{i, j}+\left(X_{0}\right)_{i, i-1}\left(A_{k-1}\right)_{i-1, j}+\cdots+\left(X_{0}\right)_{i, 1}\left(A_{k-1}\right)_{1, j}+\left(B_{0}\right)_{i, j}
\end{aligned}
$$

Let us now assume that $\left(A_{0}\right)_{p, q}=\left(A_{1}\right)_{p, q}=\left(A_{2}\right)_{p, q}=\cdots$ for all $p=i, j<q \leq$ $n$ and $1 \leq p<i, q=j$. Then

$$
\begin{aligned}
\lambda\left(A_{k}\right)_{i, j}=\lambda\left(A_{k-1}\right)_{i, j}+\left[\left(X_{0}\right)_{i, i-1}\left(A_{0}\right)_{i-1, j}+\cdots+\left(X_{0}\right)_{i, 1}\left(A_{0}\right)_{i, j}+(B)_{i, j}\right. \\
\left.-\left(A_{0}\right)_{i, j+1}\left(X_{0}\right)_{j+1, j}-\cdots-\left(A_{0}\right)_{i, n}\left(X_{0}\right)_{n, j}\right]
\end{aligned}
$$

and we conclude that $\left(A_{0}\right)_{i, j}=\left(A_{1}\right)_{i, j}=\cdots$. Inductively we obtain that

$$
\left(A_{0}\right)_{i, j}=\left(A_{1}\right)_{i, j}=\cdots \text { for all } i, j=1,2, \cdots, n
$$

and so $A_{0}=A_{1}=A_{2}=\cdots$.

Corollary 1. If $N$ is a nilpotent operator on $K$ and $X_{0}=\lambda I+N$ where $0 \neq \lambda$ $\epsilon \mathrm{C}$, then

$$
\left\|X_{0}^{n} A X_{0}^{-n}\right\| \leq M \text { for } n=0,1,2, \ldots \text { implies } A X_{0}=X_{0} A .
$$

If $K$ is finite dimensional, then the converse is also true.

Proof. Let $A_{k}=X_{0}^{k} A X_{0}^{-k}$ for $k=0,1,2, \ldots$. Then $A_{k} X_{0}=X_{0} A_{k-1}$ and the result follows from Lemma 2 by setting $B=0$.

In order to see that the converse is true if $K$ is finite dimensional, first observe that if $X_{0}$ satisfies the conclusion of the corollary, that is, $X_{0}=\lambda l+N$ for $0 \neq \lambda$ and $N$ nilpotent, then so does any operator $S X_{0} s^{-1}$ similar to $X_{0}$. Now using Jordan canonical forms it is easy to see that for any invertible operator $X_{0}$ with two or more distinct eigenvalues there is another operator $A$ satisfying $\left\|X_{0}^{n} A X_{0}^{-n}\right\| \leq M$ but $A X_{0} \neq X_{0} A$. For example, if $\mu, \lambda \neq 0$ and if 
$X_{0}=\left(\lambda l_{1}+N_{1}\right) \oplus\left(\mu l_{2}+N_{2}\right)$ on $\left[e_{n}^{(1)}\right]_{n=0}^{n} \oplus\left[e_{n}^{(2)}\right]_{n=0}^{n} 2$ where $N_{i} e_{n}^{(i)}$ equals $e_{n+1}^{(i)}$ if $n<n_{i}$ and equals 0 if $n=n_{i}$, if $A$ is defined by $A e_{0}^{(2)}=(\lambda / \mu) e_{n}^{(1)}$ and 0 otherwise, then $X_{0} A X_{0}^{-1}=(\lambda / \mu) A$. So that if $\lambda \neq \mu$ and $|\mu| \geq|\lambda|$ then $\left\|X_{0}^{n} A X_{0}^{-n}\right\|$ $\leq|\lambda / \mu|$ and $A X_{0} \neq X_{0} A$.

Lemma 3. Suppose $T \in \mathscr{B}(\mathcal{H})$ bas a lower triangular operator valued matrix on H. If $T$ commutes with $X=\left(\sum_{n=0}^{\infty} \hat{X}_{n} U_{+}^{n}\right) U_{+}$where $X_{0}=\lambda I+N$ with $0 \neq \lambda \in \mathbf{C}$ and $N$ nilpotent, then $T$ commutes with $U_{+}$.

Proof. We will show that $T$ commutes with $U_{+}$by inductively proving $T_{k, 0}=$ $T_{k+1,1}=T_{k+2,2}=\cdots$ for $k=0,1,2, \ldots$. Notice that $\left\|T_{k+j, j}\right\| \leq\|T\|$ for all $k, j=0,1,2, \ldots$.

If $1 \leq j<i$ then the $(i, j)$ entry of $T X=X T$ is

$$
\begin{aligned}
T_{i, j+1} X_{0} & +T_{i, j+2} X_{1}+\cdots+T_{i, i} X_{i-j-1} \\
& =X_{i-j-1} T_{j, j}+X_{i-j} T_{j+1, j}+\cdots+X_{0} T_{i-1, j}
\end{aligned}
$$

If $i=j+1$ we obtain $T_{j+1, j+1} X_{0}=X_{0} T_{j, j}$ and Lemma 2 implies that $T_{0,0}=$ $T_{1,1}=T_{2,2}=\cdots \cdot$. Let us now assume that $T_{l, 0}=T_{l+1,1}=T_{l+2,2}=\cdots$ for all $l \leq k$. Setting $i=j+k+2$ in (1) we obtain

$$
\begin{aligned}
& T_{k+1+j+1, j+1} X_{0} \\
& \quad=X_{0} T_{k+1+j, j}+\left[X_{1} T_{k, 0}+\cdots+X_{k+1} T_{0,0}-T_{k, 0} X_{1}-\cdots-T_{0,0} X_{k+1}\right] .
\end{aligned}
$$

Applying Lemma 2 we obtain that $T_{k+1,0}=T_{k+2,1}=\cdots$ and hence by induction $T_{k, 0}=T_{k+1,1}=\cdots$ for all $k=0,1,2, \ldots$. Thus $T$ commutes with $U_{+}$

Theorem 1. Let $V$ be a pure isometry on a Hilbert space $\mathcal{H}$, and $S \in \mathbb{B}(\mathcal{H})$ have dense range and commute with $V$. Suppose there exists a $\lambda \in \mathrm{C}$ such that $V$ factors as a product of pure isometries $V_{1}, V_{2}, \ldots, V_{n}$ and such that $S-\lambda I=$ $V_{i} S_{i}$ for each $i=1,2, \ldots, n$, where each $V_{i}$ commutes with each $S_{j}$ : Then $\{S V\}^{\prime}=\{S\}^{\prime} \cap\{V\}^{\prime}$.

Proof. It clearly suffices to prove the result for $V=U_{+}$on $\mathcal{H}=l_{+}^{2}(K)$. If $T$ commutes with $S$ and $U_{+}$then it obviously commutes with $S U_{+}$. So assume that $T$ commutes with $\mathrm{SU}_{+}$. Lemma 1 implies that $T$ has a lower triangular operatorvalued matrix on $\mathcal{H}$. Since $S$ has dense range and commutes with $U_{+}$, it follows that $\lambda \neq 0$ and that $S=\sum_{n=0}^{\infty} \hat{X}_{n} U_{+}^{n}$. We need only show that $X_{0}-\lambda I$ is nilpotent for then Lemma 3 will imply that $T$ commutes with $U_{+}$. Since $U_{+}$is one-to-one and commutes with $S$, it then follows that $T$ also commutes with $S$.

We will in fact show that $X_{0}$ has a decomposition as described in the proof 
of Lemma 2. By hypothesis $U_{+}=V_{1} V_{2} \cdots V_{n}$ and $S$ commutes with each $V_{i}$. Since $X_{0}^{*}$ is the restriction of $S^{*}$ to

$K=\mathcal{H} \Theta U_{+} \mathcal{H}=\left(\mathcal{H} \circlearrowleft v_{1} \mathcal{H}\right) J V_{1}\left(\mathcal{H} \Theta v_{2} \mathcal{H}\right) \oplus \cdots \uplus v_{1} v_{2} \ldots v_{n-1}\left(\mathcal{H} \mapsto v_{n} \mathcal{H}\right)$,

it follows that $X_{0}^{*}$ is upper triangular and hence that $X_{0}$ is lower triangula. Let $\left(x_{0}\right)_{i i}$ be the compression of $x_{0}$ to $V_{1} V_{2} \cdots V_{i-1}\left(\mathcal{H} \Theta V_{i} \mathcal{H}\right)$. If $f, g \in$ $\mathcal{H} \Theta V_{i} \mathcal{H}$ then, since $S=\lambda I+V_{i} S_{i}$, we obtain

$$
\begin{aligned}
S\left(V_{1} V_{2} \ldots V_{i-1} f\right) & =\left(V_{1} V_{2} \ldots V_{i-1}\right) S f \\
& =\lambda V_{1} V_{2} \cdots V_{i-1} f+V_{1} V_{2} \cdots V_{i-1} V_{i} S_{i} f
\end{aligned}
$$

But $\left(V_{i} S_{i} V_{1} V_{2} \cdots V_{i-1} f, V_{1} V_{2} \cdots V_{i-1} g\right)=0$, hence $\left(x_{0}\right)_{i i}=\lambda I_{i}$.

Remark 1. We remark that in general it is not always true that if $V$ is a pure isometry and if $S \in \mathfrak{B}(\mathcal{H})$ has dense range and commutes with $V$ then $\{S V\}^{\prime}=$ $\{S\}^{\prime} \cap\{V\}^{\prime}$. A counterexample is $V=U_{+}$on $l_{+}^{2}\left(C^{2}\right)$ and $S=\hat{A}$ where $A$ has eigenva lues $1 / 2$ and 1 .

3. Analytic Toeplitz operators. In this section we reformulate Theorem 1 in terms of analytic Toeplitz operators and obtain numerous consequences.

Theorem 2. Let $\phi \in H^{\infty}$ and $\phi=\chi F$ be its inner-outer factorization. If for some $\lambda \in \mathbf{C}, \chi$ factors as $\chi=\chi_{1} \chi_{2} \cdots \chi_{n}$ with each $\chi_{i}$ an inner function and $F-\lambda$ divisible by each $\chi_{i}$, then $\left\{T_{\phi}\right\}^{\prime}=\left\{T_{\chi}\right\}^{\prime} \cap\left\{T_{F}\right\}^{\prime}$.

Proof. If $\chi$ is constant, then the result is obvious since $\phi=F$. If $\chi$ is nonconstant, then as remarked earlier $T_{X}$ is a pure isometry and $T_{F}$ has dense range and commutes with $T_{\chi}$. By hypothesis there exist $g_{i} \in H^{\infty}$ such that $F(z)-\lambda=\chi_{i}(z) g_{i}(z)$. Hence $T_{F}-\lambda I=T_{\chi_{i}} T_{g_{i}}$, and of course $T_{\chi_{i}}$ commutes with $T_{g_{i}}$. Thus Theorem 1 implies $\left\{T_{\phi}\right\}^{\prime}=\left\{T_{\chi}\right\}^{\prime} \cap\left\{T_{F}\right\}^{\prime}$.

Corollary 2. Let $\phi \in H^{\infty}$ and $\phi=\chi F$ be its inner-outer factorization. If

$$
\chi(z)=z^{k} \prod_{i=1}^{\infty}\left[\frac{\left|\alpha_{i}\right|}{a_{i}} \frac{a_{i}-z}{1-\bar{a}_{i} z}\right]^{n_{i}},
$$

$a_{i} \in \mathbf{D}$ distinct, $n_{i} \leq N$, and $F(0)=F\left(\alpha_{i}\right)$ for all $i$, then $\left\{T_{\phi}\right\}^{\prime}=\left\{T_{\chi}\right\}^{\prime} \cap\left\{T_{F}\right\}^{\prime}$.

Proof. Factor $\chi^{(z)}=\chi_{1}(z) \chi_{2}(z) \cdots \chi_{N}(z)$ where each $\chi_{i}(z)$ is a Blaschke product in which distinct $\alpha_{k}$ appear at most once. Since $F(0)=F\left(a_{i}\right)$ for all $i, F-F(0)$ is divisible by each $\chi_{i}$, and Theorem 2 implies the conclusion.

Corollary 3. Let $\phi \in H^{\infty}$ and $\phi=\chi F$ be its inner-outer factorization. If $\chi(z)=((a-z) /(1-\bar{\alpha} z))^{n}, n \geq 0, a \in \mathbf{D}$ then $\left\{T_{\phi}\right\}^{\prime}=\left\{T_{\chi}\right\}^{\prime} \cap\left\{T_{F}\right\}^{\prime}$. 
Proof. Obvious by Corollary 2.

Proposition 1. Suppose $\phi \in H^{\infty}$ is such that $\phi-\phi(a)$ bas a zero of order $n \geq 1$ at $a \in \mathbf{D}$ and that there exists $\epsilon>0$ such that

$$
\left|(\phi(z)-\phi(\alpha)) /(z-a)^{n}\right| \geq \epsilon>0 \text { for all } z \in \mathbf{D}, z \neq a \text {. }
$$

Then the inner factor of $\phi-\phi(\alpha)$ is $((\alpha-z) /(1-\bar{\alpha} z))^{n}$.

Proof. The hypotheses imply that $(z-\alpha)^{n} /(\phi(z)-\phi(\alpha)) \in H^{\infty}$. Writing

$$
\left(\frac{z-\alpha}{1-\bar{\alpha} z}\right)^{n}=\frac{(z-\alpha)^{n}}{\phi(z)-\phi(\alpha)}(\phi(z)-\phi(\alpha))\left(\frac{1}{1-\bar{\alpha} z}\right)^{n}
$$

and noting that the inner-outer factorization of any function is unique, one easily concludes that the inner factor of $\phi-\phi(\alpha)$ is $((z-\alpha) /(1-\bar{a} z))^{n}$ (see p. 51 in [6]).

Remark 2. Since $\left\{T_{\phi}\right\}^{\prime}=\left\{T_{\phi-c}\right\}^{\prime}$ for any $c \in \mathrm{C}$, Corollary 3 and Proposition 1 enable one to calculate the commutant of a large class of analytic Toeplitz operators. For example, if $\phi(z)=z(\beta-z), 0<|\beta|<1$, then $\phi$ does not satisfy the hypothesis of Corollary 2, but some translate of $\phi$ satisfies Corollary 3 and one can conclude that $\left\{T_{\phi}\right\}^{\prime}=\left\{T_{z}\right\}^{\prime}$.

Corollary 4. If $\phi \in H^{\infty}$ is sucb that $\phi-\phi(a)$ bas a simple zero for some $\alpha \in \mathbf{D}$ and $|(\phi(z)-\phi(\alpha)) /(z-\alpha)| \geq \epsilon>0$ for all $z \in \mathbf{D}, z \neq \alpha$, then $\left\{T_{\phi}\right\}^{\prime}=\left\{T_{z}\right\}^{\prime}$.

Proof. Proposition 1 implies the inner factor of $\phi-\phi(\alpha)$ is $(\alpha-z) /(1-\bar{\alpha} z)$. Since $\left\{T_{(a-z) /(1-\bar{a} z)}\right\}^{\prime}=\left\{T_{z}\right\}^{\prime}$, Corollary 3 implies $\left\{T_{\phi}\right\}^{\prime}=\left\{T_{z}\right\}^{\prime}$..

Corollary 5. If $\phi \in H^{\infty}$ is univalent then $\left\{T_{\phi}\right\}^{\prime}=\left\{T_{z}\right\}^{\prime}$.

Proof. A univalent function satisfies the hypothesis of Corollary 4 for every $a \in \mathbf{D}$.

Remark 3. It is possible to give a direct proof of Corollary 5 without using Theorem 1. Suppose $\phi \in H^{\infty}$ is univalent and $T$ commutes with $T_{\phi}$. Since $\phi-$ $\phi(\alpha)$ is also univalent for each $\alpha \in \mathbf{D}, \phi-\phi(\alpha)$ has inner factor $(\alpha-z) /(1-\bar{\alpha} z)$. Now $T$ commutes with $T_{\phi}$ implies $T^{*}$ commutes with $T_{\phi-\phi(a)}^{*}$ for each $a \in \mathbf{D}$. Hence $T^{*}$ leaves invariant null $\left(T_{\phi-\phi(\alpha)}^{*}\right)=\operatorname{null}\left(T_{(\alpha-z) /\left(1-\bar{\alpha}_{z}\right)}^{*}\right)=\operatorname{span}\left(K_{\alpha}\right)$ where $K_{\alpha}(z)=(1-\bar{a} z)^{-1}$. Thus for each $\alpha \in \mathbf{D}$ there exists $\overline{\lambda(a)} \in \mathbf{C}$ such that $T^{*} K_{\alpha}$ $=\overline{\lambda(a)} K_{a}$. Since $|\lambda(z)| \leq\left\|T^{*}\right\|=\|T\|$ for each $z \in \mathbf{D}$ and

$$
\begin{aligned}
\lambda(z) f(z) & =\lambda(z)\left(f, K_{z}\right)=\left(f, \overline{\lambda(z)} K_{2}\right)=\left(f, T^{*} K_{z}\right) \\
& =\left(T f, K_{z}\right)=(T f)(z) \text { for all } f \in H^{2} .
\end{aligned}
$$


Therefore $\lambda \in H^{\infty}$ and $T$ is the analytic Toeplitz operator $T_{\lambda}$. This method of proof was first used by A. Shields and L. Wallen in [13].

Remark 4. For $\phi \in H^{\infty}$ define the cluster set of $\phi$ by

$$
C(\phi)=\left\{\lambda \in \mathbf{C}: \exists z_{n} \in \mathbf{D},\left|z_{n}\right| \rightarrow 1, \phi\left(z_{n}\right) \rightarrow \lambda\right\}
$$

then the hypothes is of Corollary 4 can be rephrased as: If $\phi \epsilon H^{\propto}$ is such that $\phi-\phi(\alpha)$ has a simple zero for some $\alpha \in \mathbf{D}$ and $\phi(\alpha) \notin C(\phi)$. A significant fact concerning the cluster set of $\phi$ is contained in the next proposition (although the result is not new [5], the proof is).

Proposition 2. The Fredholm spectrum of an analytic Toeplitz operator $T_{\phi}$ is exactly the cluster set $C_{\phi}$.

Proof. Recall that $\lambda$ is not in the Fredholm spectrum of an operator $T$ if and only if $T-\lambda$ is Fredholm, that is $T-\lambda$ has closed range and the null spaces of $T-\lambda$ and $(T-\lambda)^{*}$ are both finite dimensional. For convenience we consider $\lambda=0$. Note that $T_{\phi}-\lambda=T_{\phi-\lambda}$ is always oneto-one unless $\phi(z) \equiv \lambda$.

Suppose $0 \notin C(\phi)$ and write $\phi=B S F$ where $B$ is a Blaschke product, $S$ is a singular inner function, and $F$ is an outer function. If $S$ were nonconstant or if $B$ were an infinite Blaschke product, then one could easily find $z_{n} \in D,\left|z_{n}\right|$ $\rightarrow 1$, such that $S\left(z_{n}\right) B\left(z_{n}\right) \rightarrow 0$ contradicting $0 \notin C(\phi)$ since $F \in H^{\infty}$. Hence $S(z) \equiv 1$ and $B$ is a finite Blaschke product. Since $0 \notin \overline{\phi(D)}$ implies $\left|\phi\left(e^{i \theta}\right)\right|=$ $\left|F\left(e^{i \theta}\right)\right| \geq \epsilon>0$ almost everywhere, it follows that $F^{-1} \epsilon H^{\infty}$ and that $T_{F}$ is invertible. But $T_{B}$ is clearly Fredholm, hence $T_{\phi}=T_{B} T_{F}$ is also Fredholm.

Conversely suppose that $T_{\phi}$ is Fredholm and write $\phi=B S F$. Since $T_{\phi}$ has closed range, $\phi$ is bounded below [0], and so $T_{F}$ is invertible. If $S$ were nonconstant or if $B$ were an infinite Blaschke product then $\operatorname{dim}\left(\right.$ null $T_{B}^{*} T_{S}^{*}$ ) $=+\infty$. Since null $\left(T_{\phi}^{*}\right)=$ null $\left(T_{B}^{*} T_{S}^{*}\right)$ we must again have that $B$ is a finite Blaschke product. Thus the inner factor $B$ of $\phi$ is continuous on $T$ with $\left|B\left(e^{i \theta}\right)\right|=1$ and so $0 \notin C(\phi)$.

Remark 5. In the course of proving Proposition 2, we actually show that $T_{\phi}$ is Fredholm if and only if $\chi$ is a finite Blaschke product and $F$ is invertible in $H^{\infty}$ where $\phi=\chi F$ is the inner-outer factorization of $\phi$. In this case $T_{\chi}$ is Fredholm, and $T_{\chi}$ and $T_{\phi}$ both have the same index. The index of $T_{\chi}$ is exactly the negative of the number of zeros of $\chi$ counting multiplicity. Furthermore $\phi$ and $X$ have the same zeros. If we let $\phi_{r}$ be the restriction of $\phi$ to $C_{r}=$ $\{z \in \mathrm{C}|| z \mid=r<1\}$, then the winding number of $\phi_{r}, W\left(\phi_{r}\right)$, is the number of zeros of $\phi$ inside $C_{r}$. Since $\phi$ only has a finite number of zeros, we have $W\left(\phi_{r}\right)=$ - Index $T_{\phi}$ for $r$ sufficiently close to 1 . Equivalently Index $T_{\phi}=-\lim _{r \rightarrow 1}-W\left(\phi_{r}\right)$. If in addition $\phi$ is continuous on the unit circle, we have Index $T_{\phi}=-W\left(\phi_{1}\right)$ where $\phi_{1}$ is the restriction of $\phi$ to the unit circle. Now in terms of Fredholm 
operator Corollary 4 can be restated as follows: If $T_{\phi}$ is Fredholm operat or of index -1 , then $\left\{T_{\phi}\right\}^{\prime}=\left\{T_{z}\right\}^{\prime}$.

4. More on $\left\{T_{\phi}\right\}^{\prime}$. In this section we will completely characterize the commutant of $T_{\phi}$ for any function $\phi \in H^{\infty}$ whose inner factor is $z^{n}, n \geq 1$. Of course, analogous results also hold for any function $\phi \in H^{\infty}$ whose inner factor is $((\alpha-z) /(1-\alpha z))^{n}, n \geq 1, \alpha \in \mathbf{D}$.

Lemma 4. Suppose $T$ commutes with $T_{z^{n}}, n \geq 1$, and with $T$, where $f(z)=$ $a_{0}+a_{1} z+a_{2} z^{2}+\cdots \in H^{\infty}$. Let $p \geq 1$ be the smallest integer for wbich $a_{p} \neq 0$, and let $p=q n+r$ where $0 \leq q$ and $0 \leq r<n$. Then $T$ commutes with $T_{8}$ where

$$
g(z)=\left(f(z)-a_{0}\right) / z^{q n}=a_{p} z^{r}+\cdots \cdot
$$

Proof. Since $T_{f}$ commutes with $U_{+}=T_{z^{n}}$ we have that $T_{f}=\sum_{k=0}^{\infty} \hat{A}_{k} U_{+}^{k}$. If $q=0$ then the result follows. So assume $q \geq 1$. By the definition of $p$ we have that $A_{0}=a_{0} I, A_{1}=0, \cdots, A_{q-1}=0$. Since $T$ commutes with $T_{f}$ we obtain

$$
T\left(\sum_{k=0}^{\infty} \hat{A}_{k} U_{+}^{k}\right)=\left(\sum_{k=0}^{\infty} \hat{A}_{k} U_{+}^{k}\right) T \text { or } T\left(\sum_{k=q}^{\infty} \hat{A}_{k} U_{+}^{k}\right)=\left(\sum_{k=q}^{\infty} \hat{A}_{k} U_{+}^{k}\right) T .
$$

Hence

$$
T\left(\sum_{k=0}^{\infty} \hat{A}_{k+q} U_{+}^{k}\right)=\left(\sum_{k=0}^{\infty} \hat{A}_{k+q} U_{+}^{k}\right) T
$$

since $T$ commutes with $U_{+}$, and since $U_{+}$is isometric. But by definition of $g$ we have that $T_{g}=\sum_{k=0}^{\infty} \hat{A}_{k+q} U_{+}^{k}$

Lemma 5. Suppose $T$ commutes with $T_{z^{n}}, n \geq 1$, and with $T$, where $f(z)=$ $a_{0}+a_{r} z^{r}+\cdots+\epsilon H^{\infty}, a_{r} \neq 0,1 \leq r<n$. If $r$ divides $n$ then $T$ commutes with $T_{z^{r}}$

Proof. Since $T_{f}$ commutes with $U_{+}=T_{z^{n}}$, we have that $T_{f}=\sum_{k=0}^{\infty} \hat{A}_{k} U_{+}^{k}$ Since $r$ divides $n$, say $n=q r_{\text {, }}$ we have that $A_{0}$ is unitarily equivalent to

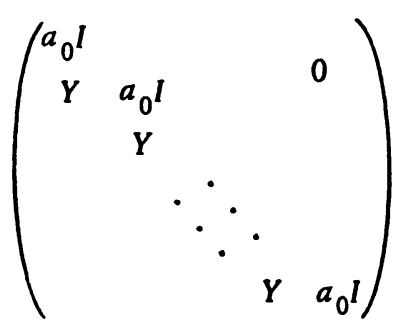


on $\sum_{l=1}^{q} \oplus \mathrm{C}^{r}$, where $Y=a_{r} I+N$ on $\mathrm{C}^{r}, a_{r} \neq 0, N$ nilpotent. Since $T$ commutes with $U_{+}=T_{z^{n}}$ we also have that $T=\sum_{k=0}^{\infty} \hat{B}_{k} U_{+}^{k}$. In order that $T$ commute with $T_{f}$ it is necessary that $B_{0}$ commute with $A_{0}$. But one easily checks that since $Y=a_{r} I+N, a_{r} \neq 0, N$ nilpotent this implies that $B_{0}$ is lower triangular on $\sum_{l=1}^{q} \oplus \mathrm{C}^{r}$, which in turn implies that $T$ is lower triangular with respect to the decomposition of $H^{2}$ for $V_{+}=T_{z} r$. Notice that

$$
T_{f}-a_{0} I=\left(\sum_{k=0}^{\infty} \hat{X}_{k} V_{+}^{k}\right) V_{+}
$$

and $X_{0}=Y=a_{r} I+N$ with $N$ a nilpotent operator. Lemma 3 now applies and we obtain that $T$ commutes with $V_{+}=T_{z}$.

Lęmma 6. Suppose $T$ commutes with $T_{z^{n}}, n \geq 1$, and with $T_{\text {, where }} f(z)=$ $a_{0}+a_{p} z^{p}+\cdots \in H^{\infty}, a_{p} \neq 0$. If $p=q n+r$ with $0 \leq q$ and $0<r<n$ then $T$ com. mutes with $T_{z}$ s where $s=$ g.c.d. $(r, n)$.

Proof. By Lemma 4, $T$ commutes with $T_{g}$ where

$$
g(z)=a_{p} z^{r}+\cdots
$$

Now if $s=r$ then $r$ divides $n$ and Lemma 5 implies that $T$ commutes with $T_{z}$. Otherwise there exist integers $t, k \geq 0$ such that $s=t r-k n$. Hence $t r=k n+s$, $0 \leq k, 0<s<n$. Since $T$ commutes with $T_{g^{\prime}} T$ also commutes with $T_{g}^{t}=T_{g} t$ where $g(z)^{t}=a_{p}^{t} z^{t r}+\cdots$ By Lemma $4, T$ then commutes with $T_{b}$ where $b^{8}(z)=$ $a_{p}^{t} z^{s}+\cdots, 0<s<n$. Since $s$ divides $n$, Lemma 5 now implies that $T$ commutes with $T_{z^{s}}$.

Theorem 3. If $\phi \in H^{\infty}$ bas inner-outer factorization $\phi=\chi F$ where $\chi(z)=$ $z^{n}, n \geq 1$, then $\left\{T_{\phi}\right\}^{\prime}=\left\{T_{z^{s}}\right\}^{\prime}$ where $s \geq 1$ is the positive integer which is maximal with respect to the property that both $z^{n}$ and $F(z)$ are functions of $z^{s}$ (equiv. alently: that $\phi$ is a function of $z^{s}$ ).

Proof. Clearly if $\chi$ and $F$ are both functions of $z^{s}$ and if $T$ commutes with $T_{z^{s}}$ then $T$ commutes with $T_{\chi}$ and $T_{F}$, and hence with $T_{\phi}$.

Now suppose that $T$ commutes with $T_{\phi}$. Corollary 3 then implies that $T$ commutes with $T_{z^{n}}$ and $T_{F}$ where $F(z)=a_{0}+a_{1} z+a_{2} z^{2}+\cdots \in H^{\infty}$. Let $s \geq 1$ be maximal with respect to the property that both $z^{n}$ and $F(z)$ are functions of $z^{s}$. Denote the sequence of integers $p \geq 1$ such that $a_{p} \neq 0$ by $p_{1}<p_{2}<p_{3}<\cdots$ Then $s=$ g.c.d. $\left(n, p_{1}, p_{2}, \cdots\right)$. Let $s_{k}=$ g.c.d. $\left(n, p_{1}, p_{2}, \cdots, p_{k}\right)$. Now suppose $p_{1}=q_{1} n+r_{1}$ with $0 \leq q_{1}$ and $0 \leq r_{1}<n$. If $r_{1}=0$ then $s_{1}=n$ and $T$ 
commutes with $T_{z_{1}}$, while if $0<r_{1}$ then Lemma 6 implies that $T$ commutes with $T_{z_{1}} s_{1}$ since g.c.d. $\left(n, r_{1}\right)=$ g.c.d. $\left(n, p_{1}\right)=s_{1}$. In either case $T$ commutes with $T_{z_{1}}$. From this one concludes that $T$ also commutes with $T_{f_{1}}$ where $f_{1}(z)=f(z)-a_{0}-a_{p_{1}} z^{p_{1}}=a_{p_{2}} z^{p_{2}}+\cdots$. Now suppose $p_{2}=q_{2} s_{1}+r_{2}$ with $0 \leq q_{2}$ and $0 \leq r_{2}<s_{1}$. If $r_{2}=0$ then $s_{2}=s_{1}$ and $T$ commutes with $T_{z^{2}} s_{2}$, while if $0<r_{2}$ then Lemma 6 implies that $T$ commutes with $T_{z_{2}}$ since g.c.d. $\left(s_{1}, r_{2}\right)=$ g.c.d. $\left(s_{1}, p_{2}\right)=$ g.c.d. $\left(n, p_{1}, p_{2}\right)=s_{2}$. From this one concludes that $T$ commutes with $T_{f_{2}}$ where $f_{2}(z)=f(z)-a_{0}-a_{p_{1}} z^{p_{1}}-a_{p_{2}} z^{p_{2}}=a_{p_{3}} z^{p_{3}}+\cdots \cdots$ Now suppose $p_{3}=q_{3} s_{2}+r_{3}$ with $0 \leq q_{3}$ and $0 \leq r_{3}<s_{2}$. If $r_{3}=0$ then $s_{3}=$ $s_{2}$ and $T$ commutes with $T_{z_{3}}$, while if $0<r_{3}$ then Lemma 6 implies that $T$ commutes with $T_{z} s_{3}$ since g.c.d. $\left(s_{2}, r_{3}\right)=$ g.c.d. $\left(s_{2}, p_{3}\right)=$ g.c.d. $\left(n, p_{1}, p_{2}, p_{3}\right)$ $=s_{3}$. Continuing in this manner we obtain that $T$ commutes with $T_{z} s_{k}$ for every k. Hence $T$ commutes with $T_{z^{s}}$.

Corollary 6. Let $\phi \in H^{\infty}$ and $\phi=\chi F$ be its inner-outer factorization with $\chi(z)=z^{n}, n \geq 1$, and $F(z)=a_{0}+a_{1} z+a_{2} z^{2}+\cdots$. If there exists an integer $p \geq 1$ such that $a_{p} \neq 0$ and g.c.d. $(n, p)=1$ then $\left\{T_{\phi}\right\}^{\prime}=\left\{T_{z}\right\}^{\prime}$.

Proof. Theorem 3 applies and the hypotheses imply that $s=1$.

Remark 6. Berkson, Rubel, and Williams [2] define an operator $A \in \mathfrak{B}(\mathcal{H})$ to be totally hyponormal if $\{A\}^{\prime}$ consists entirely of hyponormal operators. It follows that $\{A\}^{\prime}$ must be abelian. An analytic Toeplitz operator $T_{\phi}$ is totally hyponormal if and only if $\left\{T_{\phi}\right\}^{\prime}=\left\{T_{z}\right\}^{\prime}$. What analytic functions give rise to totally hyponormal analytic Toeplitz operators? Corollaries 4,5 , and 6 yield many examples of such functions. In particular $T_{\psi_{n}}$ with $\psi_{n}(z)=z^{n}(1+z), n \geq 1$, has commutant equal to $\left\{T_{z}\right\} \%$. It is interesting to note that although the commutant of $T_{\psi_{n}}$ is $\left\{T_{z}\right\}^{\prime}$, the weakly closed algebra generated by $T_{\psi_{n}}$ and $l$ is in fact smaller than $\left\{T_{z}\right\}^{\prime}[12]$. It is also interesting to note that $\psi_{n}, \psi_{n}^{2}, \psi_{n}^{3} \ldots$ all give rise to totally hyponormal Toeplit $z$ operators. In fact, using Corollary 6 it is possible to describe a large class of functions $\phi \in H^{\infty}$ such that $T_{\phi}$ and all its powers are totally hyponormal. For a related example see p. 177 of [11].

Corollary 7. If $n, k \geq 1$ are positive integers, then $\left\{T_{z^{n}}\right\}^{\prime} \cap\left\{T_{z^{k}}\right\}^{\prime}=\left\{T_{z^{s}}\right\}^{\prime}$ where $s=$ g.c.d. $(n, k)$.

Proof. Observe that if $T$ commutes with $T_{z^{n}}$ and $T_{z^{k}}$ then it also commutes with $T_{z^{n}\left(1+z^{k}\right)}$. Since $1+z^{k}$ is outer, Theorem 3 implies that $T$ also commutes with $T_{z} s$ where $s$ can be described as in the corollary.

Corollary 8. If $n, k \geq 1$ are positive integers and $0<|a|<1$, then $\left\{T_{z^{n}}\right\}^{\prime}$ $\cap\left\{T_{((a-z) /(1-\bar{a} z))^{k}}\right\}^{\prime}=\left\{T_{z}\right\}^{\prime}$. 
Proof. If $T$ commutes with $T_{z^{n}}$ and $T_{((a-z) /(1-\bar{a} z))^{k}}$ then $T$ also commutes with $T_{\phi}$ where $\phi(z)=z^{n}\left(1+((\alpha-z) /(1-\bar{\alpha} z))^{k}\right)$. Since the coefficient of $z$ in the outer part of $\phi$ is nonzero, Corollary 6 implies the result.

5. Concluding remarks. We would like to conclude this paper by raising some questions which naturally arise in our paper.

Question 1. Suppose $\phi \in H^{\infty}$ has inner-outer factorization $\phi=\chi F$. Must $\left\{T_{\phi}\right\}^{\prime}=\left\{T_{\chi}\right\}^{\prime} \cap\left\{T_{F}\right\}^{\prime} ?$

Question 2. Suppose $\phi \in H^{\infty}$ is nonconstant. Is the zero operator the only compact operator commuting with $T_{\phi}$ ?

Question 2 has an affirmative answer in case $\phi(z)=z$ [3]. In fact if $\phi$ is any inner function the answer to Question 2 is yes. Thus an affirmative answer to Question 1 implies an affirmative answer to Question 2.

Question 3. Suppose $\phi \in H^{\infty}$. Is $\left\{T_{\phi}\right\}^{\prime}=\left\{T_{l}\right\}^{\prime}$ where $I$ is some inner function of which $\phi$ is a function?

Question 4. Suppose $\phi \in H^{\infty}$. Does $\left\{T_{\phi}\right\}^{\prime} \neq\left\{T_{z}\right\}^{\prime}$ imply that $\phi$ is a function of an inner function which is not a single Blaschke factor?

An affirmative answer to Question 3 obviously implies an affirmative answer to Question 4. For a related question see E. Nordgren [11].

Question 5. Suppose $\phi \in H^{\infty}$. If $T$ commutes with $T_{\phi}$ does there exist an operator $Y$ on $L^{2}$ that commutes with $L_{\phi}$ and that leaves $H^{2}$ invariant such that $T=\left.Y\right|_{H^{2}}$ ?

Since Question 5 has an affirmative answer in case $\phi$ is an inner function, an affirmative answer to Question 3 implies an affirmative answer to Question 5. Question 5 asks whether the commutant of the subnormal operator $T_{\phi}$ can be lifted to the commutant of the minimal normal extension $L_{\phi}$. Examples are known of subnormal operators (e.g. $0 \oplus T_{z}$ ) whose commutants do not lift.

Question 6. Suppose $\left\{\chi_{a}\right\}_{a \in A}$ is a family of inner functions. Is $\bigcap_{a \in A}\left\{T_{x_{a}}\right\}^{\prime}$ $=\left\{T_{l}\right\}^{\prime}$ where $I$ is some inner function of which each $\chi_{a}$ is a function?

A preprint of J. Ball [1] of the University of Virginia establishes a strong connection between Question 4 and Questions 1 and 6 if the commutant is replaced by the set of projections in the commutant.

In this paper we have given partial answers to the above questions. For example, if the inner factor of $\phi \in H^{\infty}$ is $z^{n}, n \geq 1$, then Questions 1 through 5 have affirmative answers. However, in general, the questions seem very difficult and will certainly require techniques different from those presented in this paper.

\section{REFERENCES}

1. J. Ball, Work on a conjecture of Nordgren (preprint).

2. A. Brown, On a class of operators, Proc. Amer. Math. Soc. 4 (1953), 723-728. MR 15, 538. 
3. A. Brown and P. R. Halmos, Algebraic properties of Toeplitz operators, J. Re ine Angew. Math. 213 (1964), 89-102. MR 28 \#3350; 30, 1205.

4. E. R. Berkson, L. A. Rubel and J. P. Williams, Totally hyponormal operators and analytic functions, Notices Amer. Math. Soc. 19 (1972), A393. Abstract \#693-B11.

5. R. G. Douglas, Banach algebra techniques in operator theory, Academic Press, New York, 1972.

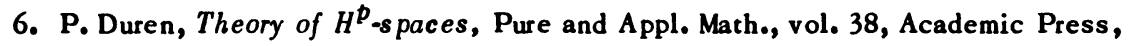
New York, 1970. MR 42 \#3552.

7. P. Fillmore, Notes on operator theory, Van Nostrand Reinhold, Math. Studies, no. 30, Van Nostrand Reinhold, New York, 1970. MR 41 \#2414.

8. P. R. Halmos, Capacity in Banach algebras, Indiana Univ. Math. J. 20 (1970), 855863. MR $42 \# 3569$.

9. P. Hartman and A. Wintner, On the spectra of Toeplitz's matrices, Amer. J. Math. 72 (1950), 359-366. MR 12, 187.

10. K. Hoffman, Banach spaces of analytic functions, Prentice-Hall Ser. in Modern Analysis, Prentice-Hall, Englewood Cliffs, N. J., 1962. MR 24 \#A2844.

11. E. Nordgren, Reducing subspaces of analytic Toeplitz operators, Duke Math. J. 34 (1967), 175-181. MR 35 \#7155.

12. D. Sarason, Invariant subs paces and unstarred operator algebras, Pacific J. Math. 17 (1966), 511-517. MR 33 \#590.

13. A. L. Shields and L. J. Wallen, The commutants of certain Hilbert space operators, Indiana Univ. Math. J. 20 (1970), 777-788. MR 44 \#4558.

DEPARTMENT OF MATHEMATICS, UNIVERSITY OF KANSAS, LAWRENCE, KANSAS 66044

DEPARTMENT OF MATHEMATICS, WAYNE STATE UNIVERSITY, DETROIT, MICHIGAN 48202 